\title{
Is Tsallis Thermodynamics Nonextensive?
}

\author{
Eduard Vives and Antoni Planes \\ Departament d'Estructura i Constituents de la Matèria, Universitat de Barcelona, \\ Diagonal 647, 08028 Barcelona, Catalonia, Spain
}

(Received 22 June 2001; published 26 December 2001)

\begin{abstract}
Within the Tsallis thermodynamics framework, and using scaling properties of the entropy, we derive a generalization of the Gibbs-Duhem equation. The analysis suggests a transformation of variables that allows standard thermodynamics to be recovered. Moreover, we also generalize Einstein's formula for the probability of a fluctuation to occur by means of the maximum statistical entropy method. The use of the proposed transformation of variables also shows that fluctuations within Tsallis statistics can be mapped to those of standard statistical mechanics.
\end{abstract}

DOI: 10.1103/PhysRevLett.88.020601

PACS numbers: 05.70.Ln, 05.20.Gg, 05.40.-a

During the past few years there has been a great deal of interest in studying nonextensive thermodynamics [1-3]. This results from the assumption of nonadditive statistical entropies and the maximum statistical entropy principle, following the information theory formulation of statistical mechanics proposed by Jaynes [4]. Indeed, besides its relevance in many nonequilibrium problems, nonextensivity is of interest for systems of particles which show longrange interactions [5], as occurs in a number of ferroic materials [6] such as ferromagnetic, ferroelastic, ferroelectric solids, and astrophysical systems [7]. Within this framework, the Tsallis statistical entropy [8] has proven to be the only nonadditive generalization of Gibbs-Shannon entropy which satisfies the following properties: (i) positivity (it takes zero value for absolute certainty), increasing monotonously with increasing uncertainty, and (ii) concavity. However, many fundamental features regarding the connection between the formulation of statistical mechanics and thermodynamics remain unclear. For instance, the identification of adequate generalized thermodynamic forces and the computation of statistical fluctuations are still controversial [9]. In this Letter we clarify such problems and provide robust arguments showing the equivalence of the present formulations of nonextensive Tsallis thermodynamics with the standard extensive equilibrium formulation.

Within the Tsallis formalism, the lack of information associated with any probability distribution $\{p(i)\}$ defined on a set of microstates $\Omega=\{i\}$ [10] is given by

$$
S_{\Omega}(\{p(i)\})=-\sum_{i \in \Omega}[p(i)]^{q} \ln _{q} p(i),
$$

where the parameter $q$, determining the degree of nonextensivity, is positive in order to ensure the concavity of $S$. The $q$-logarithmic function is defined as $\ln _{q} f=\left(f^{1-q}-\right.$ 1) $/(1-q)$. In the $q \rightarrow 1$ limit, Eq. (1) reduces to the Gibbs-Shannon entropy $S=-\sum_{i} p(i) \ln p(i)$. In order to simplify the notation, we will indicate the set $\Omega$ only when necessary. The novelty of the statistical entropy (1) is that it does not satisfy additivity. Instead, for two systems $A$ and $B$ described by independent probability distributions,

$$
S(A+B)=S(A)+S(B)+(1-q) S(A) S(B) .
$$

For a given physical system the equilibrium probability distribution $\left\{p^{*}(i)\right\}$ corresponds to the distribution that maximizes $S$ under the normalization condition $\left[\sum_{i \in \Omega} p(i)=1\right]$ and the relevant constraints imposed by the available statistical information on the system. The thermodynamic equilibrium entropy is then identified with $k S^{*}$, where $S^{*}=S\left(\left\{p^{*}(i)\right\}\right)$ and $k$ is the Boltzmann constant. In the case of an isolated (microcanonical) system no statistical information is available, and maximization of (1) leads to the equiprobability distribution on $\Omega$. Difficulties arise when trying to study nonisolated systems for which some constants of motion $X_{\alpha}(i)$ in the isolated system, such as the energy, volume, magnetization, number of particles, etc., become fixed only on average $\left(\left\langle X_{\alpha}\right\rangle\right)$. The index $\alpha$ extends over the number of such controlled observables of the nonisolated system. It is worth noting that a crucial property of such observables, required by the foundations of thermodynamics [11], is that they are additive, i.e., for two independent systems $A$ and $B$,

$$
\left\langle X_{\alpha}(A+B)\right\rangle=\left\langle X_{\alpha}(A)\right\rangle+\left\langle X_{\alpha}(B)\right\rangle .
$$

Within the Jaynes scheme, imposing knowledge of the statistical information (i.e., the controlled average values $\left.\left\langle X_{\alpha}\right\rangle\right)$ on the maximization procedure leads to the standard, canonical, grand-canonical, etc., ensembles. Two different strategies can be adopted when using the Tsallis statistical entropy [9].

(i) Unbiased averaging. - When the average quantities of the microscopic properties are defined in the standard way $\left\langle X_{\alpha}\right\rangle=\sum_{i \in \Omega} p(i) X_{\alpha}(i)$, the Legendre structure of standard thermodynamics is not recovered [9]; i.e., in equilibrium, the partition function cannot be related to a Legendre transformation of the entropy. For this reason this scheme has not been studied very much.

(ii) Biased averaging.- A Legendre structure is recovered if averages are defined as $\left\langle X_{\alpha}\right\rangle_{q}=\sum_{i \in \Omega} P_{q}(i) X_{\alpha}(i)$, 
where the averages are performed using the so-called $q$-scort probabilities: $P_{q}(i)=[p(i)]^{q} / \sum_{i \in \Omega}[p(i)]^{q}$. After maximization, when this choice is used, one recovers a fundamental thermodynamic identity which reads

$$
d S^{*}=\sum_{\alpha} y_{\alpha} d\left\langle X_{\alpha}\right\rangle_{q}
$$

where $y_{\alpha}$ correspond to the Lagrange parameters necessary for keeping the average constraints $\left\langle X_{\alpha}\right\rangle_{q}$ when performing the maximization of the statistical entropy. The physical meaning of the parameters $y_{\alpha}$ is, at present, still controversial, and the parameters are not necessarily those that control mutual equilibrium between thermodynamic systems (physical temperature, pressure, magnetic field, ...). Besides, the equilibrium statistical entropy can be expressed, in any statistical ensemble, as $S^{*}=\ln _{q} Z_{q}$, where $Z_{q}$ plays the role of the partition function and can be written as

$$
Z_{q}=\sum_{i \in \Omega} e_{q}^{-\sum_{\alpha} y_{\alpha} F\left[X_{\alpha}(i)\right]}
$$

with $F(X)=\left(X-\langle X\rangle_{q}\right) / \sum_{i \in \Omega}[p(i)]^{q}$. The $q$ exponential function is the inverse of $\ln _{q}$ and is defined as $e_{q}^{f}=$ $[1+(1-q) f]^{1 /(1-q)}$. This definition makes sense only for $1+(1-q) f>0$ and, in practice, this means that within this biased scheme only the range $0<q \leq 1$ has a physical meaning $[12,13]$.

It is worth noting that a third "averaging" procedure, consisting of using the above proposed biased averaging without normalization, has been proposed [9] to be equivalent (after suitable redefinition of the Lagrange multipliers) to this normalized biased averaging strategy.

At this point it is convenient to analyze the scaling properties of the nonextensive statistical entropy $S$ satisfying the property (2). By recursively applying Eq. (2) to $\lambda$ identical systems, one obtains

$$
S(\lambda A)=\left(\begin{array}{c}
\lambda \\
1
\end{array}\right) S(A)+\left(\begin{array}{c}
\lambda \\
2
\end{array}\right)(1-q) S(A)^{2}+\cdots+\left(\begin{array}{c}
\lambda \\
\lambda
\end{array}\right)(1-q)^{\lambda-1} S(A)^{\lambda},
$$

which can be easily expressed in the compact form:

$$
S(\lambda A)=\frac{1}{1-q}\left\{[1+(1-q) S(A)]^{\lambda}-1\right\} .
$$

Of course, for $q \rightarrow 1$ the usual scaling for extensive systems is recovered.

Let us now concentrate on the analysis of the biased scheme, and consider the statistical entropy $S^{*}$ of the equilibrium states as a function of the set of extensive quantities $\left\langle X_{\alpha}\right\rangle_{q}$. Under the assumption that expression (2) [and therefore (7)] can be applied to the equilibrium distributions and thus to $S^{*}$, we can substitute $S^{*}(A)$ by $S^{*}\left(\left\{\left\langle X_{\alpha}\right\rangle_{q}\right\}\right)$. Differentiating the expression (7) with respect to $\lambda$ and taking $\lambda=1$ we obtain

$$
\begin{aligned}
\sum_{\alpha}\left\langle X_{\alpha}\right\rangle_{q} y_{\alpha}= & \frac{1}{1-q}\left[1+(1-q) S^{*}\right] \\
& \times \ln \left[1+(1-q) S^{*}\right] .
\end{aligned}
$$

Equation (8) represents a generalization of Euler's theorem for the Tsallis nonextensive entropy. By differentiating (8) and using the thermodynamic identity (4), we deduce that

$$
\sum_{\alpha}\left\langle X_{\alpha}\right\rangle_{q} d y_{\alpha}=\ln \left[1+(1-q) S^{*}\right] d S^{*}
$$

which corresponds to the Gibbs-Duhem equation for nonextensive systems. It indicates that the Lagrange parameters $y_{\alpha}$ are not intensive variables, except for $q=1$.

The equation obtained suggests a transformation of variables that keeps the structure of the thermodynamic identity and allows one to recover the standard GibbsDuhem equation. Indeed, by defining

$$
\hat{S}^{*}=\int \frac{d S^{*}}{1+(1-q) S^{*}}=\frac{\ln \left[1+(1-q) S^{*}\right]}{1-q}
$$

and

$$
\hat{y}_{\alpha}=\frac{y_{\alpha}}{1+(1-q) S^{*}},
$$

expressions (4) and (8) can be rewritten in the form

$$
\begin{gathered}
d \hat{S}^{*}=\sum_{\alpha} \hat{y}_{\alpha} d\left\langle X_{\alpha}\right\rangle_{q}, \\
\sum_{\alpha}\left\langle X_{\alpha}\right\rangle_{q} d \hat{y}_{\alpha}=0 .
\end{gathered}
$$

Therefore, $\hat{S}^{*}$ is an extensive statistical entropy [satisfying $\left.\hat{S}^{*}(\lambda A)=\lambda \hat{S}^{*}(A)\right]$ and $\hat{y}_{\alpha}$ are the conjugated intensive variables of $\left\langle X_{\alpha}\right\rangle_{q}$. It should also be noted that the condition $q \leq 1$ (necessary for the transformation to be well defined) ensures that $S^{*}$ and $\hat{S}^{*}$ have the same concavity (thus the same maxima and minima).

The above transformation of variables (10) and (11) has partially been suggested by different authors during recent years. Tsallis has already shown the extensive properties of $\hat{S}^{*}$ [8], which in fact had been proposed several years before by Rényi [14]. The intensive character of the variables $\hat{y}_{\alpha}$ was recently pointed out by Abe et al. [12], who showed that such variables control the mutual equilibrium between thermodynamic systems. Finally, the fact that standard thermodynamics is fully recovered after the transformation of variables proposed above has also been implicitly suggested by Toral [13] within the framework of the microcanonical ensemble.

Another aspect which is still under debate refers to the calculation of statistical fluctuations within Tsallis statistics. The computation of the variances of extensive quantities $\left\langle X_{\alpha}^{2}\right\rangle$ has not been clearly solved [15] not only within the unbiased averaging scheme, but also using biased averaging. Here we provide a method of computation of fluctuations which is based on a generalization of Einstein's 
fluctuation formula and is independent of the averaging scheme. Moreover, for the case of biased averaging, the use of the transformation of variables proposed above allows standard thermodynamic fluctuations to be recovered. The method has been recently proposed for the study of fluctuations in standard extensive statistics $[16,17]$.

In order to compute fluctuations within a very general framework we must study how the distribution $p^{*}(i)$ changes under virtual displacements. Such displacements can be understood as a consequence of an internal constraint which causes the deviation of the system from the equilibrium state, or as a consequence of contact with an external bath which allows changes in parameters which would be constant under total isolation. Let us consider that such a displacement can be characterized by a multidimensional parameter $\kappa$ which takes values on a continuous set $\mathcal{R}$ and vanishes at equilibrium. We define the set of functions $\left\{p^{*}(i \mid \kappa)\right\}$ as the displaced (conditional) probability distributions for a given $\kappa$ obtained after maximization of (1). Note that $p^{*}(i \mid 0)=p^{*}(i)$.

The key point for characterizing fluctuations is to consider the joint probability distribution $p(i, \kappa)$ defined on the enlarged set $\Omega \times \mathcal{R}$. This means that we are assuming that $\kappa$ is a fluctuating variable and that the system is described by simultaneously specifying $i$ and $\kappa$. The problem now is to determine $p(i, \kappa)$. Using the same philosophy used for the determination of equilibrium distributions $p^{*}(i \mid \kappa)$, we propose to generalize the maximum statistical entropy principle to the determination of $p^{*}(i, \kappa)$ on $\Omega \times \mathcal{R}$. This means maximizing

$S_{\Omega \times \mathcal{R}}(\{p(i, \kappa)\})=-\int_{\mathcal{R}} \sum_{i \in \Omega}[p(i, \kappa)]^{q} \ln _{q} p(i, \kappa) d \kappa$,

where we have assumed that the measure of the lack of information on $\Omega \times \mathcal{R}$ is also given by Tsallis entropy [18].

Taking into account the mathematical definition of the conditional probability $p(i, \kappa)=p(\kappa) p^{*}(i \mid \kappa)$ and using the standard property $\ln _{q} f g=\ln _{q} f+\ln _{q} g+(1-$ q) $\ln _{q} f \ln _{q} g$ and the normalization condition for $p^{*}(i \mid k)$, Eq. (14) can be written as

$$
S_{\Omega \times \mathcal{R}}=\int_{\mathcal{R}}[p(\kappa)]^{q} S_{\Omega}^{*}(\kappa)+S_{\mathcal{R}}(\{p(\kappa)\}),
$$

where $S_{\Omega}^{*}(\kappa)=S_{\Omega}\left(\left\{p^{*}(i \mid \kappa)\right\}\right)$ is nothing more than the thermodynamic entropy of the virtually displaced system. Therefore, the problem of determining the distribution of fluctuations reduces to the maximization of the functional (15) with respect to $p(\kappa)$ under the normalization condition $\int_{\mathcal{R}} p(\kappa) d \kappa=1$. This yields

$$
p^{*}(\kappa)=\frac{e_{q}^{S^{*}(\kappa)}}{\int_{\mathcal{R}} e_{q}^{S^{*}(\kappa)} d \kappa} .
$$

Since the $e_{q}$ function converges to the standard exponential function in the limit $q \rightarrow 1$, the above equation just reduces to the well-known Einstein formula in the extensive limit. This probability distribution for fluctuations $p^{*}(\kappa)$ is, therefore, the most probable (or the least biased) distribution of the fluctuating parameter $\kappa$. Also note that formula (16) can be deduced in a nonrigorous way (as done in many statistical mechanics textbooks in the case of extensive statistics) by inversion of the generalized Boltzmann formula for the microcanonical ensemble: $S^{*}=\ln _{q} W$, where $W$ is the number of states in $\Omega$.

Taking into account the fact that $S^{*}(\kappa)$ is maximum at $\kappa=0$ we can expand it in powers of $\kappa$ as

$$
S^{*}(\kappa)=S^{*}(0)+\frac{1}{2}\left(\frac{\partial^{2} S^{*}}{\partial \kappa^{2}}\right) \kappa^{2}+\ldots
$$

with $\left(\partial^{2} S^{*} / \partial \kappa^{2}\right)<0$. Substituting in (16), we find that small $\kappa$ fluctuations are Gaussian distributed according to

$$
p^{*}(\kappa) \sim \exp \left\{-\frac{1}{2} \frac{-\left(\frac{\partial^{2} S^{*}}{\partial \kappa^{2}}\right)}{1+(1-q) S^{*}(0)} \kappa^{2}\right\} .
$$

This formula, as well as (16), is independent of the different averaging procedures explained in the introduction. It shows that the probability distribution for fluctuations of measurable quantities depends on $q$ as well as on the entropy of the reference equilibrium state around which fluctuations take place.

In order to compute the variance $\left\langle\kappa^{2}\right\rangle$ the two averaging schemes discussed above can be considered. Nevertheless, if $\kappa$ is a macroscopic quantity, the unbiased scheme seems more appropriate. The two choices yield very similar results in the limit of small fluctuations. If we use unbiased averaging, the variance is given by

$$
\left\langle\kappa^{2}\right\rangle=-\left[1+(1-q) S^{*}(0)\right]\left(\frac{\partial^{2} S^{*}}{\partial \kappa^{2}}\right)^{-1},
$$

whereas in the case when we use the biased averaging procedure, one gets the same result, but it is divided by $q$ (the $q$-scort probability of a Gaussian distribution is also Gaussian). It is worth noting this within the two interpretations, as $q<1$ fluctuations are larger than those corresponding to the extensive case $q=1$.

It is also interesting to note that the transformation of variables (10) and (11) suggested in the previous section allows standard thermodynamics to be recovered within the biased scheme, and can be used to rewrite Eq. (16) in terms of $\hat{S}^{*}$. Using the fact that $S^{*}$ and $\hat{S}^{*}$ have the same concavity, a straightforward calculation yields

$$
p^{*}(\kappa)=\frac{e^{\hat{S}^{*}(\kappa)}}{\int_{\mathcal{R}} e^{\hat{S}^{*}(\kappa)} d \kappa}
$$

which is the standard Einstein formula, and the variance transforms into

$$
\left\langle\kappa^{2}\right\rangle=\left(\frac{\partial^{2} \hat{S}^{*}}{\partial \kappa^{2}}\right)^{-1}
$$

Therefore the transformation of variables allows not only standard thermodynamics to be recovered but also allows the same statistical fluctuations. For instance, a simple calculation for the energy fluctuations $\Delta E$ from 
this expression gives

$$
\left\langle\Delta E^{2}\right\rangle=-\left(\frac{\partial E}{\partial \hat{\beta}}\right),
$$

where $\hat{\beta}=\beta /\left[1+(1-q) S^{*}\right]$ is the intensive physical inverse temperature, and $\beta$ is the Lagrange parameter associated with $\langle E\rangle_{q}$.

In summary, we propose that Tsallis statistics, within the biased averaging scheme, can be mapped into standard thermodynamics. This is done by a suitable transformation of variables, which appears after the derivation of the generalized Gibbs-Duhem equation resulting from the scaling properties of Tsallis entropy. The intensive generalized forces in such a framework arise naturally from the transformation of variables. In addition, the standard equilibrium fluctuation-dissipation theorem is also recovered. This indicates that, besides the thermodynamic equivalence, statistical fluctuations also behave according to standard statistical mechanics. Moreover, this ensures the equivalence of the equilibrium response functions.

This work has received financial support from the CICyT (Spain), Project No. MAT2001-3251, and from the CIRIT (Catalonia), Project No. 2000GR00025. We acknowledge fruitful discussions with our colleagues, J. Casademunt, T. Castán, Ll. Mañosa, J. Ortín, and J. M. Sancho.

[1] R. Salazar and R. Toral, Phys. Rev. Lett. 83, 4233 (1999); B. P. Vollmayr-Lee and E. Luijten, Phys. Rev. Lett. 85, 470
(2000); R. Toral and R. Salazar, Phys. Rev. Lett. 85, 471 (2000).

[2] A. K. Rajagopal and S. Abe, Phys. Rev. Lett. 83, 1711 (1999).

[3] T. Yamano, Eur. Phys. J. B 18, 103 (2000).

[4] E. T. Jaynes, Phys. Rev. 106, 620 (1957).

[5] S. A. Cannas and F. A. Tamarit, Phys. Rev. B 54, R12661 (1996).

[6] V. K. Wadhawam, Introduction to Ferroic Materials (Gordon and Breach, Amsterdam, 2000).

[7] D. F. Torres, H. Vucetich, and A. Plastino, Phys. Rev. Lett. 79, 1588 (1997).

[8] C. Tsallis, J. Stat. Phys. 52, 479 (1988).

[9] C. Tsallis, R.S. Mendes, and A. R. Plastino, Physica (Amsterdam) 261A, 534 (1998).

[10] For the sake of simplicity and without loss of generality, we consider $\Omega$ as a discrete set of states.

[11] See, for instance, R. Giles, Mathematical Foundations of Thermodynamics (Pergamon, Oxford, 1964), Chap. 3.

[12] S. Abe, S. Martinez, F. Pennini, and A. Plastino, Phys. Lett. A 281, 126 (2001).

[13] R. Toral, cond-mat/0106060.

[14] A. Rényi, Probability Theory (North-Holland, Amsterdam, 1970).

[15] F. Jedrzejewski, cond-mat/0103386.

[16] A. Caticha, in Proceedings of the 20th International Workshop on Bayesian Inference and Maximum Entropy Methods Gif-sur-Yvette, France, 2000 (math-ph/0008017) (to be published).

[17] A. Planes and E. Vives, J. Stat. Phys. (to be published).

[18] A generalization of such an assumption could be done by assuming that the nonextensivity parameter $q$ depends on $\kappa$. 